\title{
Service Federation in Virtual Organizations
}

\author{
L. M. Camarinha-Matos ', H. Afsarmanesh ${ }^{2}$, E. C. Kaletas ${ }^{2}$, T. Cardoso ${ }^{1}$ \\ ${ }^{1}$ New University of Lisbon, Quinta da Torre - 2825 Caparica, Portugal, cam@uninova.pt \\ ${ }^{2}$ University of Amsterdam, Kruislaan 403, 1098 SJ Amsterdam, The Netherlands
}

\begin{abstract}
The practical implantation of the concept of dynamic virtual enterprise is still far from expectations due to a number of factors such as the lack of appropriate interoperable infrastructures and tools, lack of common ontology, and the socio-organizational difficulties. However, the creation of industry clusters supported by advanced information and communication tools can meanwhile provide a basis for the rapid creation of dynamic virtual enterprises in response to the market opportunities. A federated service management approach is introduced in this context and its application to the tourism industry is discussed. Finally, the support for the aggregation of simpler services into value-added services, implemented by distributed business processes within different organizations, is presented.
\end{abstract}

Key words: virtual organization, service federation, virtual enterprise, industry cluster.

\section{INTRODUCTION}

Virtual organization benefits and support technologies. There is already abundant literature about the potential advantages brought in by virtual organizations and virtual enterprises, and a lot of recent proposals address more advanced dynamic cooperative networked organizations. The idea of highly dynamic organizations, that form themselves according to the needs and opportunities of the market and remain operational as long as these opportunities persist, suggests a number of benefits such as:

- Agility: the ability to recognize, rapidly react and cope with the unpredictable changes in the environment in order to achieve better responses to opportunities, shorter time-to-market, and higher quality with less investment. The composition of a VE is determined by the need to associate the most suitable set of skills and resources contributed by a number of distinct individual organizations. When and if necessary, the VE can reorganize itself by adding / expelling some members or by dynamically re-assigning tasks or roles to its members.

- Complementarity: enterprises seek for complementarities (creation of synergies) that allow them to participate in competitive business 
opportunities and new markets.

- Achieving dimension: especially in the case of SMEs, being in partnerships with others allows them to achieve critical mass and appear in the market with a larger "visible" size.

- Resource optimization: smaller organizations sharing infrastructures, knowledge, and risks.

- Innovation: being in a network opens the opportunities for the exchange and confrontation of ideas, the basis for innovation.

A large number of R\&D projects try to establish some technological foundations for the support of Virtual Enterprises /Virtual Organization (VE/VO) [6]. Relevant examples can be found in the NIIIP program in US, the ESPRIT and IST programmes in Europe (e.g. projects such as PRODNET II, VEGA, X-CITTIC, VIVE, etc.), or inter-regional cooperation programmes such as the IMS (e.g. projects such as GLOBEMAN, GNOSIS, GLOBEMEN, etc.) and INCO (e.g. MASSYVE). Many of these development efforts were concentrated on the design and development of infrastructures and basic VE/VO support functionalities. But only a few of these initiatives correspond to horizontal developments, aimed at establishing the base technology, tools and mechanisms, while most others correspond to vertical developments, addressing certain needs of specific sectors such as the manufacturing, agribusiness, tourism, etc. Although it is natural that in the early phases of a new area, considerable effort is devoted to the basic infrastructures, the lack of a common and widely accepted reference model and infrastructure has forced the vertical development projects to design and implement their own infrastructures, deviating resources from their main focus.

On the other hand, the emergence of a number of standards and technologies represent potential enabling factors, such as for instance:

- Open interoperable underlying network protocols (TCP/IP, CORBAIIOP, HTTP, RMI, SOAP),

- Open distributed object oriented middleware services (J2EE Framework, CORBA Framework, ActiveX Framework),

- Standardised modelling of business components, processes and objects (EJBs, OAG and OMGs Business Objects and Components),

- Business Process Modelling Tools and Languages (UML, UEML, WfMC XML-based Business Language)

- Open and standard business process automation and Workflow Management Systems (WfMC, OMG-JointFlow, XML-WfMC standards, many commercial products),

- Standard interfacing to federated multi-databases (ODBC, JDBC),

- Intelligent Mobile Agents (FIPA, OMG-MASIF, Mobile Objects),

- Open and standard distributed messaging middleware systems (JMS, MS-Message Server, MQSeries, FIPA-ACC),

- XML-Based E-Commerce Protocols (BizTalk, CBL, OASIS, ICE, 


\section{RosettaNET, OBI, WIDL),}

- Web Integration Technologies (Servlets, JSP, MS-ASP, XSL).

However, most of these technologies are in their infancy and under development, requiring considerable effort to implement and configure comprehensive VE/VO support infrastructures.

Therefore, although the advantages of the Virtual Enterprise are well known at the conceptual level [9], [6], the practical implantation is still far from the expectations, except for the more stable, long-term networks or supply chains. Nevertheless, the potential agility of a VE in terms of fast reaction to business opportunities is certainly a desirable feature in a scenario of fast changing market conditions; but the early phase of VE planning and creation is still a difficult one that needs to be adapted even by advanced and competitive enterprises. Some of the obstacles include the lack of appropriate support tools, namely for partners search and selection, VE contract bidding and negotiation, competencies and resources management, well-established distributed business process management practices, task allocation, performance assessment, inter-operation and information integration protocols, etc. Further problems include the lack of common ontologies, and the proper support for socio-organizational aspects e.g. lack of a culture of cooperation, the time required for trust building processes, need for BP reengineering and training of people, etc. Furthermore, the fast evolution of the information technologies often represents a disturbing factor for non-IT companies.

Industry / service clusters as a basis for VE creation. The creation of long term clusters of industry or service enterprises represent an approach to overcome these obstacles and can support the rapid formation of VE / VO according to the business opportunities. The concept of cluster of enterprises, which should not be confused with a VE, represents a group or pool of enterprises and related and supporting institutions that have the potential and the will to cooperate with each other through the establishment of a long-term cooperation agreement. Buyer-supplier relationships, common technologies, common markets or distribution channels, common resources, or even common labor pools are elements that typically bind the cluster together. This is not a new concept as a large number of related initiatives have emerged during the last decades, namely in Europe and USA [4]. But the advances in information and communication technologies now bring new opportunities to leverage the potential of this concept, namely by providing the adequate environment for the rapid formation of agile virtual enterprises. For each business opportunity found by one of the cluster members, a subset of the cluster enterprises may be chosen to form a VE for that specific business opportunity. In this perspective, the expected competitive advantage of cooperative development of products and services becomes a more relevant tie among the cluster members. The more frequent 
situation is the case in which the cluster is formed by organizations located in a common region, although geography is not a major facet when cooperation is supported by computer networks.

The cluster enterprises are normally "registered" in a directory, where their core competencies are "declared". Based on this information, the VE initiator / creator, which is usually a member of the cluster enterprises, can select partners when a new business opportunity is detected. Clearly, several VEs can co-exist at the same time within a cluster, even with some members in common. A cluster represents a long-term organization and therefore presents an adequate environment for the establishment of cooperation agreements, common infrastructures and ontologies, and mutual trust, which are the facilitating elements when building a new VE. The concept of cluster is evolving in parallel with the emergence of other forms of relationships, such as "communities of practice" or "virtual communities", representing a general trend to the emergence of a kind of "society of relationships". The cluster does not need to be a closed organization; new members can adhere but they have to comply with the general operating principles of the cluster. Similarly, for the formation of a VE, preference will be given to cluster members but it might be necessary to find an external partner in case some skills or capacities are not available in the cluster. The external partner will naturally have to adhere to the common infrastructure and cooperation principles. In addition to enterprises, a cluster might include other organizations (such as research organizations, sector associations, etc.) and even free-lancer workers. The establishment and management of clusters through adequate infrastructures represent therefore an important support for the creation of agile virtual enterprises (Fig. 1).

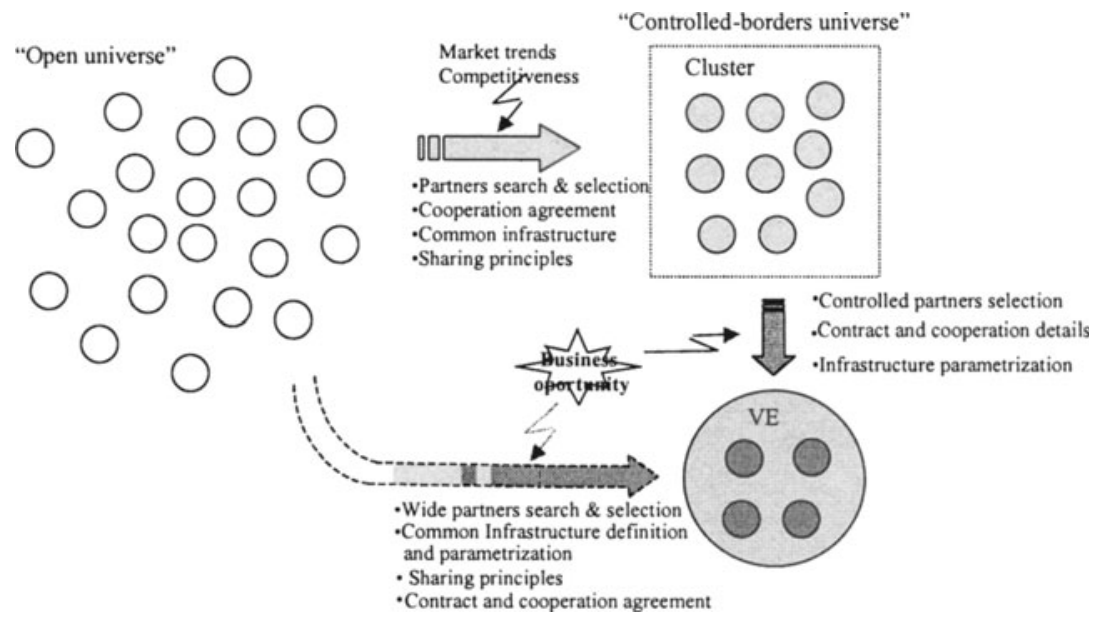

Figure 1. Two approaches for VE formation

This idea of using a cluster as the basis for the formation of virtual 
enterprises has been identified in other research works such as the COSME/VIRPLAS [10] or VIRTEC [5] projects. These projects have identified some of the major characteristics and needs of cluster management, but did not introduce the necessary IT infrastructure and support tools.

From a regional perspective, a well-managed cluster may offer the opportunity to combine the necessities of both "old" and "new" economies, and form a sustainable environment. The ICM (Industry Cluster Management) concept can support the exploitation of local competencies and resources by an agile (and fast) configuration of the most adequate set of partners for each business opportunity and therefore extending the scope of intervention of manufacturing companies into the services area. Furthermore, the local clusters can gather and empower a unique set of competencies tailored to regional culture and local customers' preferences, allowing a concerted offer of cooperation to global companies. In this way, members of the local industry cluster can play an important role in the customization and final assembly of products to local markets even though the basic components may be produced elsewhere. Therefore, in a time of tough competition provoked by the globalization, the organization and effective management of local industry or service enterprises clusters, focused on the characteristics of SMEs, provides a promising approach for regional sustainability. In addition to the mentioned benefits of cooperation on dynamic VE/VO, there is also the opportunity to share experiences and costs in the learning process of introducing new IT within an industry cluster, and to reduce the risk of failure.

Federated virtual markets. The concept of federation can support advanced management strategies for the services offered by a cluster. The federation mechanisms which have been developed in different areas such as information management (federated information management [1] [8]), multiagent systems (federation of agents [11] [3]), or services management (services federation [12][2]) represent approaches to support the interoperation among heterogeneous, autonomous and geographically distributed entities through the definition of a set of common principles and mechanisms to allow a harmonized access to services and information, independently of the diversity of their sources.

Common features among the various materializations of the federation concept are the support for cooperation among distributed, possibly heterogeneous, and autonomous entities (for example, information resources or service sources) without the need for the clients of information / services to care about the communication details. In a federated system different authorities are in charge of different parts of the system and preserve their autonomy. The local autonomy of entities, which establishes access rights to local resources, is subject only to the agreements reached by the entities with 
other authorities. These agreements, although they may be seen as a restriction of each entity's autonomy, are required for cooperation. Therefore, the involved entities have to negotiate terms and conditions of their interoperation with other entities.

When applied to the management of a cluster, this concept allows a vision of the various cluster members as service providers that, independently of the way their services are implemented or located, make them accessible in a kind of controlled "virtual market". A client such as a VE creator can "shop" in this market for the best set of services to satisfy the needs of a given business opportunity. The service federation infrastructure shall provide the basic mechanisms for service registration, maintenance and removal, mechanisms for transparent (remote) access to services according to some agreed access rules, mechanisms to define and maintain these access rules to the services. Moreover, because the services in a service federation can actually represent any available sources, different federations besides a service federation can be established. For instance, a natural means of providing information management support to service federations can be realized by coupling the service federation with an information federation, which is established through federating a set of existing information resources within the cluster, even building on top of the agreements reached for the service federation.

This paper, in this direction, addresses the development of a federated approach to service management with a particular focus on the support to the creation of valued-added services.

\section{GENERAL ASPECTS IN SERVICE FEDERATION}

One of the obvious components of a cluster management system is a directory where the cluster members are registered and their competencies / skills and capacities are declared. This directory is therefore a set of member profile records. Considering that competencies of cluster members are represented (to some extent) by services (service functions), this directory shall include a catalog of services (Fig. 2).

Due to the members autonomy (and legacy) there might be a large heterogeneity / diversity in the way services are implemented. However, in order to facilitate service selection ("shopping") and utilization, a common service interface needs to be agreed among the cluster members and, in case of legacy implementations service adapters have to be developed. A service interface can be decomposed in two parts: service specification descriptor and service invocation wrapper (or proxy), i.e. the service API.

The service specification describes the characteristics of the service such as service name and identifier, version, functionality, $\mathrm{I} / \mathrm{O}$ specifications, applicability conditions, access rights, etc. Even temporary announcements 
of service providers can be supported through this component; for instance, a "hotel reservation service" could announce here a special offer for the just married couples. When a client is looking for a specific service in the catalog, the search criteria are expressed against the properties (attributes) of the service specification component. The service invocation wrapper, on the other hand, describes the programming interface of the service, that is, for instance the methods available, their parameters and return values, etc. It acts as a proxy to the actual service, providing transparent access to the service by hiding the implementation details, such as remote invocation, security and communication mechanisms. A client (application) will get a copy of this wrapper that will be linked to the application memory space to be used for the remote (transparent) invocation of the service.

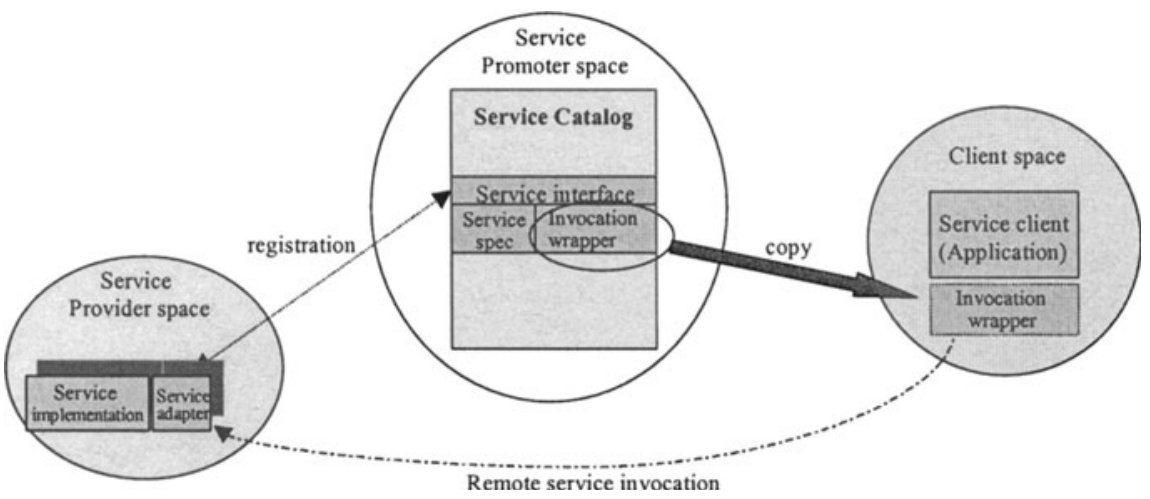

Figure 2. Services registration and invocation

From a computational point of view, the cluster can be seen as a network comprising one (or more) cluster management node(s) (or cluster promoter node(s) from a service market perspective) responsible for the catalog, and a number of member nodes representing the cluster members where the actual implementations of services may be installed (and running). Cluster members advertise their services by registering them in the cluster service catalog.

It is important to notice that the same service type can have a number of different implementations (given by different members of the federation). However a common interface has to be guaranteed (by the service providers) in order to facilitate the identification and use of these independently developed services. For this purpose a common ontology and common functional interface rules have to be agreed among the cluster members.

It shall be mentioned that several VE projects have tried to adopt / combine different standards such as EDIFACT, STEP or UDDI in order to eliminate the ontological mismatch among members of a VE. But in spite of these efforts it is difficult to have general-purpose solutions because:

- for different classes of information handled in a cooperation process there are no standards (e.g. quality-related information); 
- EDIFACT or STEP cover wide application areas and therefore for any practical application subsets have to be selected and agreed among partners.

Although difficult to be solved in general terms, the problem is more tractable in a smaller (closed) universe as the one represented by a cluster. Common rules and principles can be agreed by the initial members of the cluster. Future members, in order to join the cluster, are required to accept those principles (part of the adhesion contract). As the cluster envisions a long-term relationship (in opposition to a single cooperation opportunity), the required investment / adaptation may be affordable and give the cluster true agility whenever opportunities for cooperation arise. The implementation of a harmonized representation of services in the service catalog does not necessarily mean that all cluster members have access to all services all the time. Service providers shall keep their autonomy and the right to specify whom and under which conditions, has access rights to their registered services. Therefore there is a need for an access rights manager component (Fig. 3) allowing the definition and validation of access rights. For example, a service provider can specify that a given service description is available for lookup only to a specific set of service requesters.

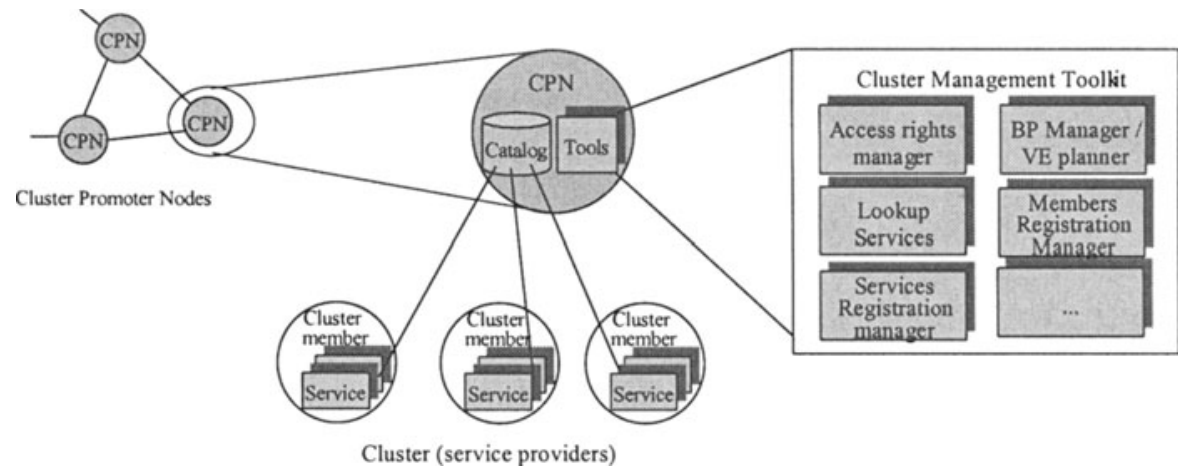

Figure 3. Cluster management functionalities

A service can be represented as an object class including a number of attributes and methods. For instance, a "hotel reservation service" could include:

Attributes:

- Service provider id

- Hotel category

- Location

- Other attributes

- Access rights list
Methods:

- Check_availability

- Check_prices

- Make_reservation

- Cancel_reservation.

When a business opportunity is found by a member of the cluster, this member assumes the role of broker (or VE initiator). This broker will typically elaborate a plan of activities (business process - BP) that are 
required to satisfy the business goal. In order to perform these activities, the broker, using a $\mathrm{BP}$ manager component, may search and select a number of service methods from the catalog. When service methods are assigned to the various steps of the BP, this BP may in fact become a distributed BP, as different parts of the process are performed by different service providers. The selection of a suitable set of service methods for a particular BP (in other words the creation of the VE) is a very important functionality that, in addition to the type of service required, has to take into account a number of other factors such as: performance history of the service provider (e.g. how reliable it is), compatibility (joint performance) between different service providers, visibility rules / access rights, and other specific requirements.

Therefore, the service catalog component shall offer intelligent search / selection filtering lookup functionalities, based on the service attributes, to assist the activity of the broker.

Higher level or value added services (VAS) might be created by a composition of different low-level services available in the catalog. Therefore, a service provider might be a service client as well as he can look for and select services from the catalog to compose his VAS. A new VAS can be registered in the catalog in the same way as the basic service. In section 4 a more detailed description of this process is presented.

In addition to the basic elements described there is a need for additional functionalities such as a members certification function (to perform an assessment of partners and how they behaved in past cooperation relationships), service assessment or certification function (handling issues such as reliability of the service, compliance with the common interface specifications, performance, etc., i.e. Quality of Service functionality), etc.

\section{AN EXAMPLE - TOURISM INDUSTRY}

Similar to the manufacturing industries, the need to remain competitive in the open market also forces the service providing companies to seek "world class" status. In the case of Tourism industry, companies remain focused on their core competencies, while realize the need to look for alliances, when additional skills / resources are needed to fulfill business opportunities. Cooperation among the actors / entities in Tourism industry is not a new phenomena. For instance, travel agencies typically offer aggregated or valueadded-services (VAS) composed of components supplied by a number of different organizations. But to provide an on-line value-added-service for "booking a complete journey plan" that may include several means of traveling, several hotel bookings, car rentals, leisure tour bookings, etc., a networked cooperation must exist among many different organizations [2]. Fig. 4 shows an example of a possible business process for a traveling package where the various activities are supported by a number of services 
provided by different organizations.

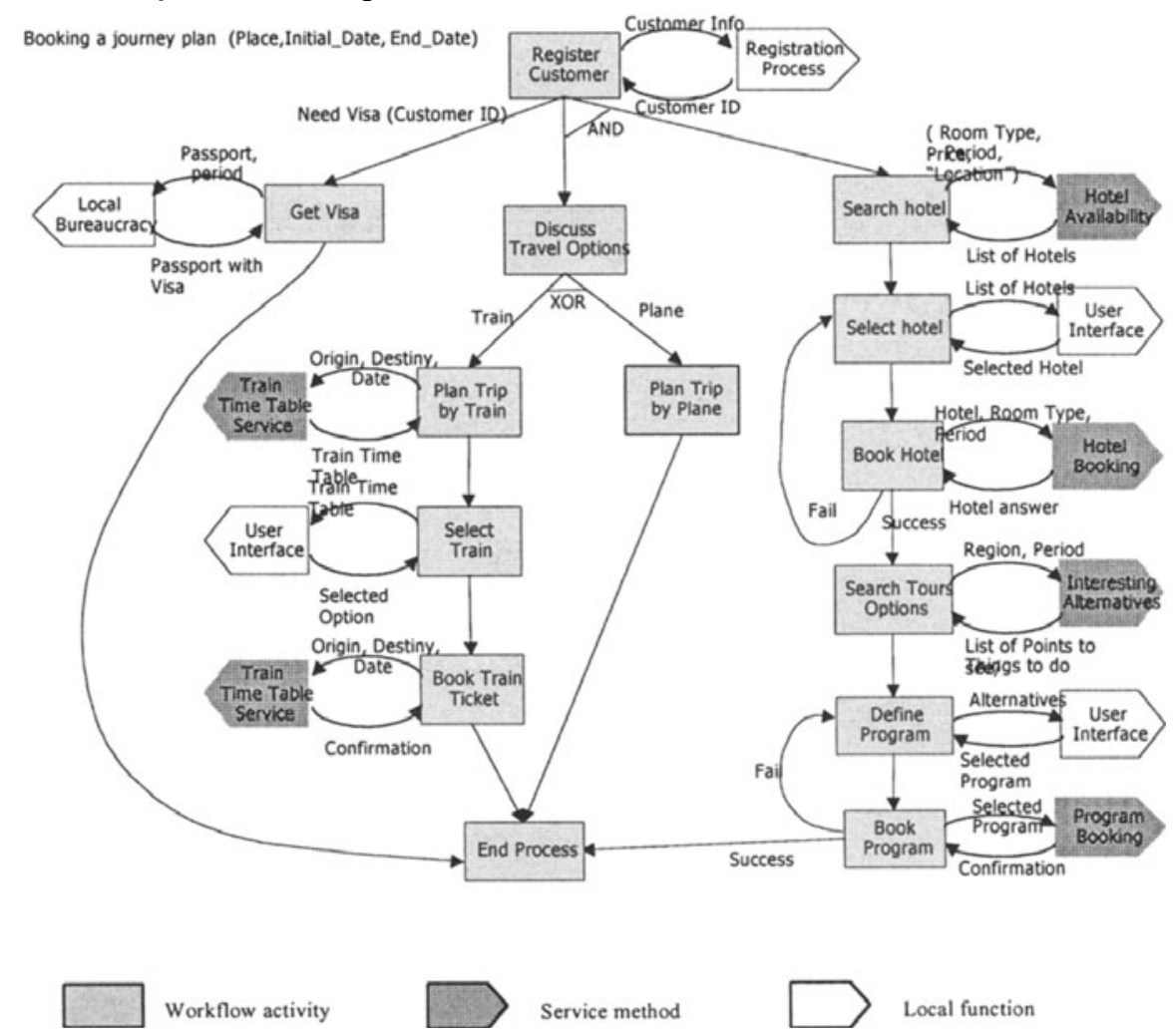

Figure 4. Example of value added service in tourism

Clustering is a typical phenomenon in this sector, especially at regional level. Regional tourism promotion organizations try to offer an integrated view of the local resources (local cluster promoter node). In order to reduce the fragmentation and dispersion of information some trends to form networks of clusters are emerging. One example is the enjoyeurope.com initiative.

Following the general principles described above, the IST FETISH project is developing a computational infrastructure to support the tourism industry in Europe. The main goals pursued in this project are: (i) to increase the availability of tourism-related services via a mechanism of services federation; and (ii) to improve the interoperation among providers and clients, taking advantage of the new facilities provided by Internet. The intention is to promote the development (and sharing) of new tourism-related services, and the affiliation of organizations to a network of service federations. For example, if a travel agency wants to offer a very specific holiday package, the proposed infrastructure which is being developed in Java/JINI, is intended to provide a set of application tools to support it in a flexible way, combining different tourism-related services offered by 
different organizations.

In order to fulfill this goal, the adopted approach considers a threelayered architecture as presented in Fig. 5. It is assumed that the members of this federation (clients and service providers) will work cooperatively through the Internet, based on the interoperation mechanisms supported by the Jini technology. The layers defined in this architecture are the following: the Service layer, the Directory layer, and the VAS Support layer.

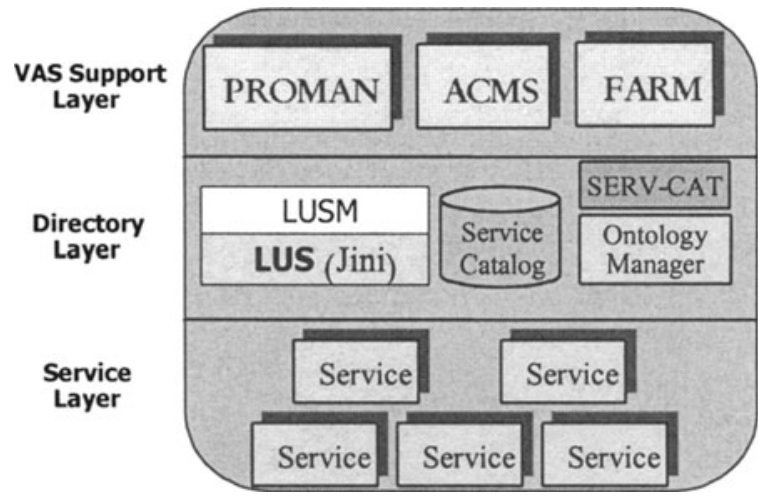

Figure 5. Three-layer architecture components

The Service layer contains the tourism services developed by the members of the federation. These services are publicized to the entire federation (e.g., a hotel reservation service, a rent-a-car service, etc.) or only to the members of a subgroup defined within a cluster. The Directory layer supports the procurement of the tourism services (advanced lookup service) implemented in the federation. This layer expands the Lookup Services (LUS) hierarchy, which is the core component of the Jini environment, by adding a specific component (the LUS Manager - LUSM, developed by SUN Spain) to handle the issues related to the service management needs. The Ontology Manager, developed by IASIS Italy, provides a common tourism ontology and mechanisms and rules for registering new service interfaces. The interfaces are stored in the SERV-CAT module developed by the University of Amsterdam. The VAS Support layer is intended to offer high-level applications that can be executed on top of the LUSM hierarchy offering advanced functionalities to the federation. Some of the main applications at this level are:

(i) The Process Management system (PROMAN) developed by Uninova, that aims at supporting the distributed business process (DBP) creation and management, as it will be described in next section.

(ii) The ACcess Manager System (ACMS), which serves as the entry gateway for the end users and service providers to FETISH, where some kind of human intervention is required. In this case, ACMS shall present an integrated user interface for certain important offered capabilities of 
FETISH, such as the "New service interface definition request" and the "Quality control and service operation maintenance".

(iii)Federated Access Rights Manager (FARM), developed by the University of Amsterdam. This module allows the definition and validation of access rights defined at the level of service proxy in the general context of FETISH as well as within the Virtual Enterprises dynamically created. For instance, a service provider can specify that a given service proxy can be available for lookup only to a specific set of service requesters or only to specific VE members. Therefore, this module must support the proper configuration of the access rights. Furthermore, the proper filtering and validation of access rights have to be accomplished at the moment in which the lookup process is being performed. This can be realized in two ways:

- There is a FARM module next to every LUSM node in the FETISH system, and each FARM node manages the access rights definitions for the proxies stored only in that LUSM node. In this case, after a lookup to the LUSM, the resulted proxies (or their IDs) are passed to the FARM for the validation of access rights against the requester, and only the validated proxies are returned to the requester. In this case, FARM must work in close cooperation with the LUSM.

- There is a single FARM module within the FETISH system to manage the access rights for all FETISH service proxies. In this case, the ACMS coordinates the activities within the FETISH system, and after a lookup passes the proxies returned by the LUSM network to the FARM for the evaluation of access rights. Then the validated proxies are returned back to the user.

The tourism services can be provided in different levels. The lower level contains the basic tourism services, which represent atomic services created by a tourism operator. On top of this level, it is possible to create another level in which the basic services are used as components of a Value Added Service. Let us consider that we can create (sub)VASs to be used as part of another VAS. In this way, we can foresee that it is possible to create an unlimited number of different levels of services. It is important to emphasize that all services are handled inside the federation in the same way, irrespective of the level they belong to.

In order to illustrate how this system works, let us consider that "Hotel Palma" is a member of one tourism cluster. Such a node wants to develop a new service called "HotelReservation". Therefore, the first step is to check if there is a specification for the service type in the Service Catalog. If the specification is found, the new service has to comply with that description. In case it is a new service type, it is necessary to create a new service specification and submit it to the ACMS module for possible registration in the Service Catalog (Figure 6a). Please note that a service specification contains the information that characterizes the service, such as: service 
identifier, input and output parameters, additional attributes, etc. In other words it is an abstract representation of the service.

After that, the service is developed by Hotel Palma. This development is an internal task that has to be performed by the node itself. Now, in order to put the "HotelReservation" service available to the federation, the Hotel Palma has to develop a "wrapper" or API that contains all methods and mechanisms that support the activation of the "HotelReservation", and register such a wrapper in the Service Catalog. In Figure $6 \mathrm{~b}$, for illustrative purposes only, this wrapper is called Wrapper_HRP ("HotelReservation Palma"). Please note that there might be several implementations of a service type (by different service providers). For instance, the service "HotelReservation" can be implemented by any of the hotels that belong to a given cluster. In this case, the only restriction applied to the different implementations is the compatibility with the interface registered for that service in the Service Catalog. For each implementation a wrapper (API) has to be registered in the Catalog.

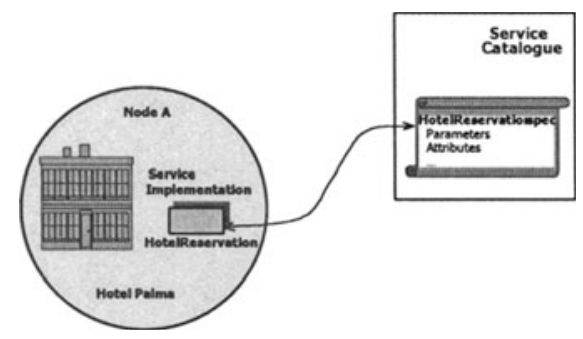

Figure 6a. Registering a Service Interface

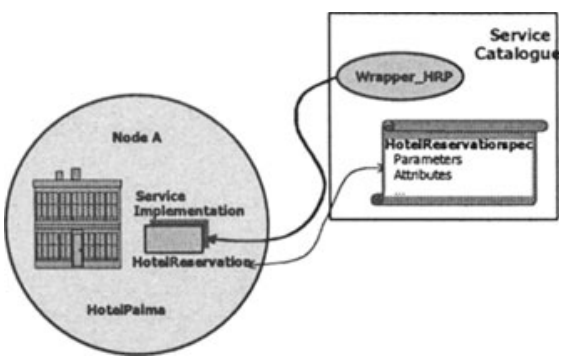

Figure $6 b$. Registering a Service Wrapper

In addition, let us suppose that a travel agency "Paradise Tours" needs to reserve a room in Hotel Palma. If Paradise already knows that the "HotelReservation" service is available from hotel Palma, Paradise gets a copy of the "Wrapper_HRP" and uses it to make the reservation (Fig. 7). If Paradise Tours does not know about the availability of this service, it has to browse through the service catalog to eventually find that service interface.

The common interfaces defined for services are stored in the Service Interface Definitions Catalog (SERV-CAT). As mentioned earlier, common interfaces facilitate the service selection and utilization in a cluster of autonomous members. When a service provider is willing to develop and register a new service, it will first look in the SERV-CAT for the best suitable service interface among the available interfaces. Once an appropriate interface is found, the service provider will implement this interface to develop its service.

The SERV-CAT is implemented using the Oracle DBMS. The relational database model structures of SERV-CAT to store the service interfaces definitions correspond to the Java language structures. For instance, the types (classes and interfaces) defined within the SERV-CAT are grouped 
under packages. A type has a number of members, namely fields and methods. Each member has a type and a default value, while methods have parameters and return types. The parameter and return types of methods, however, are stored as XML files in the SERV-CAT.

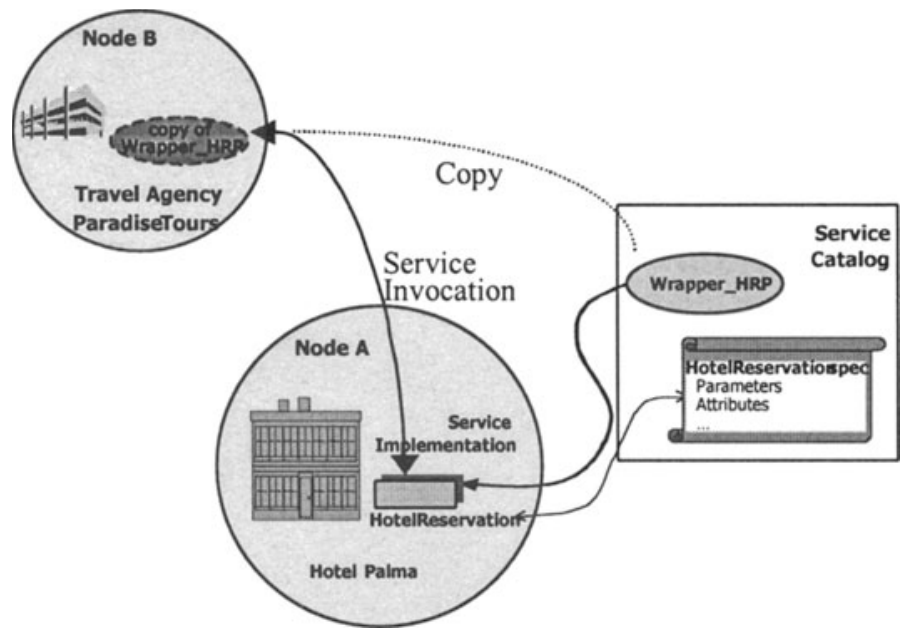

Figure 7. Using a tourism service

In order to separate the application logic from the presentation logic, an application server is developed, together with a Java FetishTypes package representing the data structures of SERV-CAT as Java classes to be used by the application server and client. The application server, SERV-CAT-AS, provides the functionality necessary for the client applications to browse and manipulate the catalog. The SERV-CAT-AS is an RMI server, which is continuously running and waiting for client requests. When a request arrives, it accesses the Oracle database using JDBC, and converts the result set into the corresponding FetishTypes objects, and returns it back to the client. The architecture for the SERV-CAT and its related tools is given in Fig. 8.

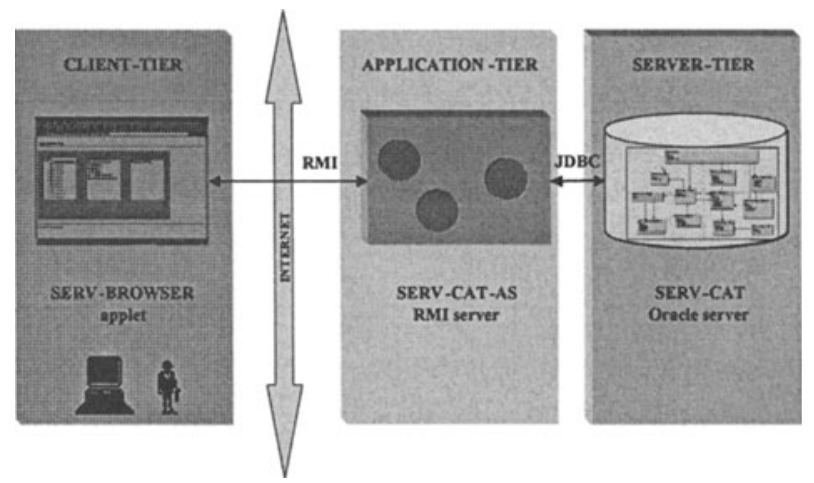

Figure 8. The SERV-CAT architecture

Other examples of capabilities to be supported by ACMS are:

1.New service specification definition request. Clearly, "new service type 
definitions" require human intervention for intelligent decision making e.g. by a committee, that translates the request into "new concept definitions" to be further modelled and stored in the common ontology. The ontology definitions will be then translated into "new catalog definitions" to be submitted for storage within the Service Catalog.

2. Quality control and service operation maintenance. A procedure needs to be followed to handle for instance the users reports and/or complains on the service quality or availability. This procedure clearly requires human attention and intervention for intelligent decision-making. This will partially involve the directory administrator to monitor and /or control the proper availability and operation of services through the LUSM.

Besides the dynamic consortia established to execute each VAS, longterm cooperation agreements are also sometimes established among subsets of enterprises within a cluster or network of clusters to jointly support certain services. When a customer contacts a travel agency, he/she frequently notices that the agency shows clear preferences for certain specific providers, probably those that are involved in long-term cooperation agreement (formal or tacit), or those with whom the agency had good past experiences in other previous cooperation processes. Some other types of consortia may have a more permanent "institutional" organization, with or without a common ownership, for example the Best Western hotel chain. The three types of consortia (temporary, long-term, and permanent) require flexible common mechanisms namely in terms of access rights definition and control that are provided by FARM.

\section{VALUE ADDED SERVICES}

As illustrated by the tourism sector, the aggregation of services into higher level or valued-added services is an important process in a clusterbased service market. Travel agencies or tourism intermediaries typically offer aggregated or value-added services composed of components supplied by a number of different organizations. The "materialization" of such a value-added service involves a process - business process (BP) - requiring the invocation and coordination of other services that may be provided by different members of the cluster. In such a case of distributed execution the $\mathrm{BP}$ becomes a distributed business process (DBP). The contributing members can therefore form a temporary organization or virtual enterprise. The DBP has to be properly managed in order to guarantee that it is successfully performed. This process management activity involves many issues including:

(i) The definition of the DBP and its structural decomposition in terms of BPs, sub-BPs, and activities; 
(ii) The distribution of such BPs/ activities among the VE members;

(iii) The execution of each $\mathrm{BP} /$ activity by the VE members; and

(iv) A supervision process capable of handling the problems detected during the execution of BPs/ activities that may affect the performance of the whole VE.

Proper coordination policies supported by flexible coordination mechanisms are necessary to ensure the cooperation among partner enterprises. A typical approach, usually found in the workflow management systems, and the work on the coordination languages, focuses on the separation between the coordination and computing (or service methods processing). At the same time, the sharing and exchange of information in a cooperative virtual organization is always inter-related to both the business processes running at each enterprise, as well as the distributed business processes that run at different sites. Therefore information management aspects cannot be independent of the business process coordination issues.

In order to support these requirements a Process Management System (PROMAN) based on the concept of federated market of services is developed by the Robotics and CIM group of the New University of Lisbon / Uninova. The Fig. 9 presents the architecture of PROMAN, which is composed of three modules, namely: the Business Processes Administrator (BPA), the Business Process Editor (BPED), and the Business Process Executor (BPEX). The BPA is the PROMAN interface to the external entities (other components of the cluster management system) and the manager of BPED and BPEX. Both BPED and BPEX provide functionalities to the BPA, respectively "edition" and "execution/monitoring" functions. The BPED is a graphical editor tool that supports the BP edition-related needs in PROMAN. The BPEX is a multi-thread execution system that supports the execution and monitoring-related needs in PROMAN.

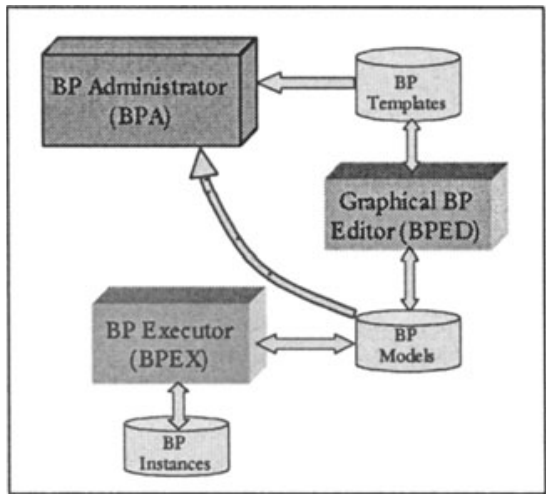

Figure 9. General structure of PROMAN

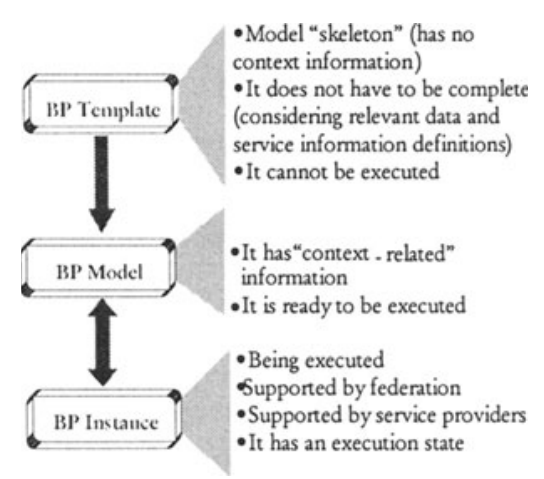

Figure 10. BP templates, models, and instances

PROMAN handles three main types of entities: BP templates, BP models, and BP instances (Fig. 10). A BP template represents a "skeleton" that can be used to support the creation of a BP Model, which represents a VAS. The 
main difference between a template and a model is that the latter contains "context-related" information that allows its execution. Besides that, some construction rules can be relaxed in the templates, since they will be further extended and analyzed before being executed. Each execution of a BP model is represented by a $\mathrm{BP}$ instance.

BP templates can be shared with the entire cluster or a subset of partners. This means, a cluster node can create a BP template and store it in a kind of "central repository of templates", from where it is downloaded and used by any other allowed cluster node. Similarly, a BP model (as a VAS implementation) may be put available to the entire cluster (or network of clusters), a subset of this cluster, or even to outside clients. A BP model is created and registered following the procedure already described for services registration. This means that the BP interface is registered in the Service Catalog, the "BP proxy" is registered in the LUSM network, and such a BP must be ready to be executed when remotely activated by a client. The main difference between the publication of BP template and BP model is that the template itself is available to be downloaded from the "central repository of templates", whilst the model is available to be only executed remotely. In other words, while a template itself is offered to the community becoming a "shared property" among the cluster members, a model is developed and remains as a "property" of its developer.

Both BP templates and models are created using a graphical language (Fig. 11) provided by BPED (Fig. 12). The main entities used in BP Templates and Models are the following: Begin/End, Activity, Transition, Service (remote, local, or sub-BP), Relevant data, Split, and Join. Begin/End are the symbols used only to mark both the starting and the ending points of a template/model. An activity represents a task to be performed, which can be a single (atomic) task or a complex one (implemented as a sub-BP). A single task is represented by the basic service methods, which are invoked directly from the service providers, via the proxies registered in the LUSM network. A complex activity invokes, in fact, another BP. This mechanism supports a hierarchical utilization of the BPs. A transition is the connector that links two activities. Local functions correspond to proprietary functionalities provided by the VAS implementer. The entire set of transitions defines a path to be followed when a BP model is executed. A relevant data set is used to handle the flow of information among the activities that belong to a BP model. Splits/Joins are used to define an additional logic mechanism to work together with the transitions, in order to define the path to be followed during the execution of a BP model.

Some characteristics of a BP model are the following:

$\circ$ It is composed of a set of activities linked by transitions following a parallel and/or sequential flow;

- Each activity invokes a service method (basic, sub-BP or a local function); 
- The transitions can be conditioned and temporized;

- Loops and cycles are allowed in a BP model;

- It can be partially defined, which means, it is not previously completed in terms of activities, services and data involved in. These can be dynamically provided during the instantiation of this model;

- Supports the definition of exception handling procedures.

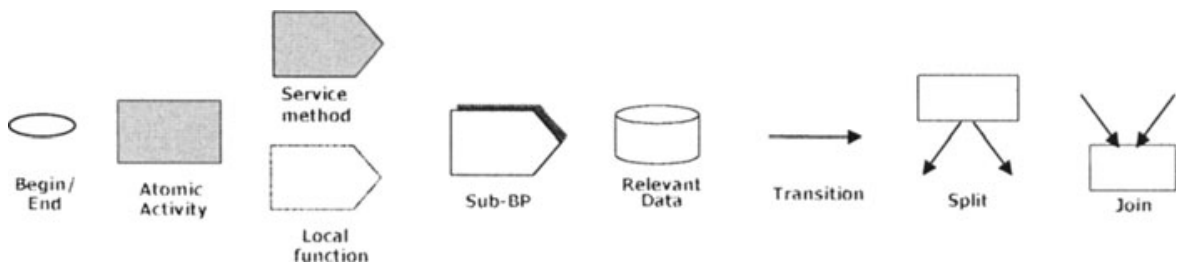

Figure 11. Graphical BP definition language

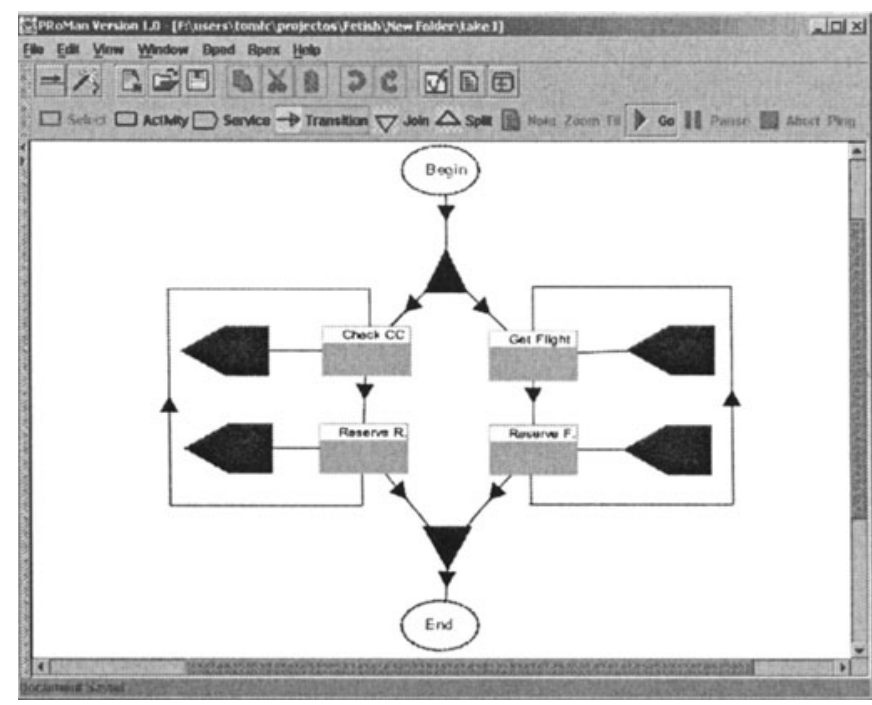

Figure 12. Example of BPED use

The main functionalities of the BPA component include:

- Support the management of BP templates, models, and instances;

- Save/Load of BP templates and models;

- Upload/Download the BP templates to/from the Service Catalog;

- Find service interfaces;

- Find service proxies (in interaction with the LUSM module);

- Register VASs interfaces in the Service Catalog;

- Register VASs proxies in the Service Catalog;

- Provide a persistent storage for each BP instance;

- Support the recovery process of BP instances;

- Support dynamic changes in BP models (temporary and permanent). 
BPED functionalities include:

- Edit BP templates/models: Insert, eliminate, select, copy, move entities, etc.;

- Graphical facilities: Visualization resources (Grid, Zoom, etc.); Configuration of BP services icon;

- Validate BP templates/models (graphics, syntax, and semantics);

- Trigger the execution of BP models;

- Support a "constrained edition" of BP models (i.e., partially "lock" the edition of a BP model, which has one BP instance being executed).

Finally, the list of BPEX functionalities includes:

$\circ$ Load \& Parsing BP models;

- Validate BP models (check syntax and semantics);

- Creation of the BP instance;

- Accept a remote activation of a VAS;

- Execute services (remote or local invocations);

- Manage exceptions;

- Optional pre-validation of models;

- Support Dynamic Changes: temporary \& permanent; a "pause" mechanism during the execution of a BP instance; interaction with $\mathrm{BPED}$ in order to support the changes;

- Save/Load of BP instance status information, events information;

- Color-based representation of the BP instance status;

- Support the intervention from a human operator.

It shall be noted that although a BP model assigns a service method to each activity, it might happen that this service is not available at the moment of its invocation. This might be due to a temporary network problem or because the service provider, as an autonomous entity, decided to change its policy. Therefore, before actually invoking services, BPEX has to check their availability (e.g. through the leasing mechanism of Jini) or the network connections. In order to reduce the number of exceptions requiring specific recovery, a BP model may associate a list of alternative service methods to each activity. PROMAN extends the coordination model first developed in the PRODNET project [7] by considering a service federation model.

\section{CONCLUSIONS}

An industry cluster, when supported by adequate management functions, forms a proper environment for facilitating the rapid creation of dynamic virtual enterprises in response to business opportunities.

A federated services approach as a basis for a virtual market of services represents a flexible solution for managing the services offered by the various members of an industry cluster while preserving their privacy and autonomy. 
On top of the service federation infrastructure, a complementary functionality is offered for value-added services creation and the corresponding distributed business process modeling.

Further work is being carried out on the integration of the value-added services definition and the federated access rights management mechanisms.

Acknowledgements. The authors thank the European Commission and IFIP for the partial support for this work and their partners of the FETISH-ETF, MASSYVE and COVE projects for their valuable contributions.

\section{References}

1. Afsarmanesh, H.; Tuijnman, F.; Wiedijk, M.; Hertzberger, O. - Distributed schema management in a cooperation network of autonomous agents, Proceedings of DEXA'93, Prague, Czech Republic, 1993.

2. Afsarmanesh, H.; Camarinha-Matos, L.M. - Future smart organizations: A virtual tourism enterprise, Proceedings of WISE 2000- $I^{\text {st }}$ ACM/IEEE International Conference on Web Information Systems Engineering, Vol. 1 (Main Program), pp 456-461, IEEE Computer Society Press, ISBN 0-7695-0577-5, Hong Kong, 19-20 June 2000.

3. Beasley, M.; Cameron, J.; Girling, G.; Hoffner, Y.; Linden, R.; Thomas, G. - Establishing Co-operation in Federated Systems, Systems Journal, Vol. 9, Issue 2, Nov. 1994.

4. Bergman, E. M.; Feser, E. J. - Industrial and regional clusters: Concepts and comparative applications, www.rri.wvu.edu/WebBook/Bergman-Feser, 2000.

5. Bremer, C. F.; Mundim, A.; Michilini, F.; Siqueira, J.; Ortega, L., 1999 - A Brazilian case of VE coordination, in Infrastructures for Virtual Enterprises - Networking Industrial Enterprises, L.M. Camarinha-Matos, H. Afsarmanesh (Ed.s), Kluwer Academic Publishers, ISBN 0-7923-8639-6, Oct 1999.

6. Camarinha-Matos, L. M., and Afsarmanesh, H., 1999 - Infrastructures for Virtual Enterprises - Networking Industrial Enterprises, Kluwer Academic Publishers, ISBN 07923-8639-6, Oct 1999.

7. Camarinha-Matos, L. M.; Lima, C., 1999 - PRODNET coordination module, in [6].

8. Garita, C.; Ugur, Y.; Frenkel, A.; Afsarmanesh, H.; Hertzberger, L.O, 2000 - DIMS: Implementation of a Federated Information Management System for PRODNET II, Proceedings of 11 th International Conference and Workshop on Database and Expert Systems Applications - DEXA '2000, London, England.

9. Goranson, H.T., 1999 - The Agile Virtual Enterprise - Cases, metrics, tools. Quorum Books, ISBN 1-56720-264-0.

10. Molina, A.; Flores, M.; Caballero, D., 1998 - Virtual enterprises: A Mexican case study, in Intelligent Systems for Manufacturing, L.M. Camarinha-Matos et al. (Eds.), Kluwer Academic Publishers, ISBN 0-412-84670-5.

11. Osorio, A.; Camarinha-Matos, L.M. - A federated multi-agent infrastructure for concurrent engineering (CIM-FACE), Studies in Informatics and Control, Vol. 5, N. 2, June 1996, pp.143-156.

12. SUN, 1999 - JINI Technology Architectural Overview, Jan 1999, http://www.sun.com/jini/whitepapers/architecture.html. 